\title{
SURFACE RUNOFF MODEL IN GERA SOFTWARE: PARALLEL IMPLEMENTATION AND SURFACE-SUBSURFACE COUPLING
}

\author{
V. KRAMARENKO ${ }^{1,2}$ AND K.NOVIKOV ${ }^{3 *}$ \\ ${ }^{1}$ Nuclear Safety Institute of the Russian Academy of Sciences, Moscow, Russia. \\ ${ }^{2}$ Sechenov University, Moscow, Russia. \\ ${ }^{3}$ Marchuk Institute of Numerical Mathematics of the Russian Academy of Sciences, Moscow, Russia. \\ *Corresponding author. E-mail: konst.novikov@gmail.com
}

\section{DOI: 10.20948/mathmontis-2020-49-6}

Summary. In this article we present a mathematical model used for surface runoff simulation in GeRa software. The model is based on diffusive wave approximation for the shallow water equations with Manning formula for flow velocity estimation. It is implemented using INMOST software platform for parallel mathematical modeling. Parallel efficiency of the model implementation is adressed for some widely used verification benchmarks. We also present surface-subsurface coupling approach used in GeRa software and discuss practical aspects of the nonlinear solver.

\section{INTRODUCTION}

Surface water is one of the key components of the hydrologic budget of the watershed. Thus computational efficiency of the surface runoff model implementation as well as effective surface-subsurface coupling become of great concern to hydrologic modeling software developers. Considering multiprocessor architecture of the modern computers it is natural to use distributed approach for mathematical models implementation.

Surface runoff model in GeRa software [1] based on 2D diffusive wave approximation of the shallow water equations [2] coupled with subsurface flow model based on 3D Richards equation with consideration of fluid and medium compressibility [3] is implemented numerically using finite volume discretization method with two-point flux approximation and Newton iterations as a nonlinear solver. Surface and subsurface models are coupled by firstorder exchange flux [4], [5].

Recently, a large number of different highly efficient computational codes for groundwater modelling have appeared [6][7][8][9] and parallelization of different aspects of this process remains challenging [10], [11], [12], [13]. The GeRa software was developed taking into account the necessity of massive parallel calculations [14]. Now this code is used for highperformance modelling of real objects [15]. Parallelization of surface flow modelling unit is required for the integration with the rest part of the GeRa software. Coupled surfacesubsurface model parallelization is carried out using INMOST platform for distributed mathematical modeling [16]. Moreover, feature set of INMOST includes tools for automatic differentiation for residual vector and jacobian matrix construction for nonlinear solver.

In this article, we address parallel efficiency of the surface runoff GeRa model in conjunction with groundwater flow model. The serial version of the model was previously discussed in [17]. Here we address the parallel implementation of the model. Coupled model is tested and verified using benchmarks presented in [4]. The solution obtained using GeRa software is compared to numerical results of other surface-subsurface simulators such as ATS [11], GEOtop [17], [18], HGS [19], Parflow [20], InHM [21], [22], An and Yu model [23],

2010 Mathematics Subject Classification: 76S05, 65C20, $65 Y 05$.

Key words and Phrases: Surface water, groundwater, surface-subsurface interaction, parallel computations. 
OpenGeoSys [24], [25], Cast3M [26], CATHY [27], MIKE-SHE [28].

We also address numerical issues caused by discontinuous surface-subsurface flux close to zero surface water levels.

\section{SURFACE RUNOFF MATHEMATICAL MODEL}

Let's consider a domain $\Omega \in \mathbb{R}^{3}$ with boundary $\partial \Omega=\Gamma_{s} \cup \Gamma_{g}$, where $\Gamma_{s}$ is surface boundary and $\Gamma_{g}$ is subsurface boundary. $\Omega$ corresponds to a geological domain with $\Gamma_{s}$ being the land surface. Surface runoff model is applied in the two-dimensional domain $\Gamma_{s}$. In GeRa the model is based on diffusive wave approximation of shallow water equations and Manning formula for friction slopes [19]:

$$
\frac{\partial h_{s}}{\partial t}-\nabla \cdot\left(K_{s} \nabla H_{s}\right)=q-q_{s s}
$$

where

$$
K_{s}=\frac{h_{s}^{5 / 3}}{v \sqrt{\left|\nabla H_{s}\right|}}
$$

and $h_{s}=h_{s}(x, t)$ is the unknown surface water depth, $H_{s}(x, t)=h_{s}(x, t)+z(x), v$ is the Manning's roughness coefficient, $q$ is the precipitation rate, $q_{s s}$ is the surface-subsurface flux density. We refer to $K_{s}$ as a surface conductivity coefficient.

Two types of boundary conditions are considered on the boundary $\partial \Gamma_{s}$. The first one is critical depth boundary condition [19]:

$$
-K_{s} \nabla H_{s} \cdot \mathbf{n}=\sqrt{g h_{s}^{3}},
$$

and the second one is homogeneous Neumann boundary condition:

$$
-K_{s} \nabla H_{s} \cdot \mathbf{n}=0
$$

where $\mathbf{n}$ is outward unit normal vector, $g$ is the gravity acceleration.

To model groundwater flow we use modified Richards equation for variably saturated media with consideration of fluid and medium compressibility in domain $\Omega$ [3]:

$$
\frac{\partial \theta\left(h_{g}\right)}{\partial t}+S s_{\text {stor }} \frac{\partial h_{g}}{\partial t}-\nabla \cdot K_{g} \nabla\left(h_{g}+z\right)=0,
$$

where $\theta$ is the water content, $h_{g}$ is the pressure head, $S=S\left(h_{g}\right)=\frac{\theta}{\theta_{s}}$ is the saturation, $s_{\text {stor }}$ is the specific storage, $K_{g}=K\left(h_{g}\right)$ is the hydraulic conductivity, $\theta_{s}$ is the maximum (saturated) water content.

Water content $\theta$ is associated with pressure head by van Genuchten model [29]: 


$$
\theta=\left\{\begin{array}{l}
\theta_{r}+\frac{\theta_{s}-\theta_{r}}{\left(1+\left|\alpha h_{g}\right|^{n}\right)^{m}}, h_{g}<0 \\
\theta_{s}, h_{g} \geq 0
\end{array}\right.
$$

where $\theta_{r}$ is residual water content, $\alpha$ and $n$ are model parameters, $m=1-1 / n$. Hydraulic conductivity is approximated using Mualem's model [30]:

$$
K_{g}=K_{s a t} S_{e}^{0.5}\left(1-\left(1-S_{e}^{\frac{1}{m}}\right)^{m}\right)^{2}
$$

where $K_{\text {sat }}$ is saturated conductivity, $S_{e}=\frac{\theta-\theta_{r}}{\theta_{s}-\theta_{r}}$ is the effective saturation.

The following Neumann type boundary conditions are set on $\partial \Omega$ :

$$
-K_{g}\left(h_{g}(x, t)+z\right) \nabla h_{g}(x, t) \mathbf{n}=\left\{\begin{array}{l}
q_{s s}, x \in \Gamma_{s}, \\
0, x \in \Gamma_{g} .
\end{array}\right.
$$

Here $\boldsymbol{n}$ is an outward normal vector to the boundary.Thus on $\Gamma_{s}$ the flux is defined by surface-subsurface water interaction and is zero on the rest of the boundary.

Surface-subsurface coupling approach is based on first-order exchange coefficient [31] (i.e. flux density is proportional to difference between surface water depth and subsurface pressure head):

$$
q_{s s}\left(h_{s}, h_{g}\right)=\left\{\begin{array}{l}
\frac{K_{s s}}{d}\left(h_{s}-\overline{h_{g}}\right) \text { for }\left(h_{s}>0,-\infty<\overline{h_{g}}<+\infty\right) \text { and }\left(h_{s}=0, \overline{h_{g}}>0\right), \\
0 \text { for }\left(h_{s}=0, \overline{h_{g}} \leq 0\right)
\end{array}\right.
$$

where $K_{s s}$ is bottom sediments conductivity, $d$ is the bottom sediments layer thickness, $\overline{h_{g}}$ is the limited groundwater pressure head. The latter is determined by the following formula with small positive $\epsilon_{g}$ :

$$
\overline{h_{g}}=\frac{1}{2}\left(h_{g}-d+\sqrt{\left(h_{g}+d\right)^{2}+\epsilon_{g}^{2}}-\epsilon_{g}\right)
$$

This expression is used to provide nonlinear solver convergence and smoothly approximate the following value:

$$
\widehat{h_{g}}=\max \left\{h_{g},-d\right\} \text {. }
$$

As one can get negative $h_{s}$ during the nonlinear solver iterations, definition of expression (10) should be extended for $h_{s}<0$ : 


$$
q_{s s}\left(h_{s}, h_{g}\right)=\left\{\begin{array}{l}
\frac{K_{s s}}{d}\left(h_{s}-\overline{h_{g}}\right) \text { for }\left(h_{s}>0,-\infty<\overline{h_{g}}<+\infty\right) \text { and }\left(h_{s} \leq 0, \overline{h_{g}}>h_{s}\right), \\
0 \text { for }\left(h_{s} \leq 0, \overline{h_{g}} \leq h_{s}\right) .
\end{array} .\right.
$$

This definition means that water flow cannot have a downward direction (from surface to subsurface) when there is no water on the surface.

Equation (12) is discontinuous with respect to both arguments for $h_{s}=0, h_{g}<0$. The discontinuity may result in nonlinear solver oscillations near the surface-subsurface flux discontinuity line. To overcome this problem, we use modified formula (smoothed) for surface-subsurface flux. To provide further details we first decompose the range of $h_{s}$ and $h_{g}$ into 3 subdomains (see fig. 1). We use the domain $A$ as an interface between domains $B$ and $C$ and smooth the flux function in it. The following expression is used for surface-subsurface flux which is continuously differentiable with respect to both arguments for $h_{s} \geq 0$ except the square domain $0 \leq h_{s} \leq \varepsilon, 0 \leq h_{g} \leq \varepsilon$, where it is discontinuous along the $h_{s}=h_{g}$ segment,

$$
q_{s s}\left(h_{s}, h_{g}\right)=\left\{\begin{array}{l}
\frac{K_{s s}}{d}\left(h_{s}-\overline{h_{g}}\right),\left(h_{s}, h_{g}\right) \in C, \\
\frac{K_{s s}}{d}\left(-\frac{1}{\varepsilon^{2}} h_{s}^{3}+\frac{1}{\varepsilon} h_{s}^{2}+\left(h_{g}-\varepsilon\right) \frac{1-\cos \frac{\pi h_{s}}{\varepsilon}}{2}\right),\left(h_{s}, h_{g}\right) \in A, . \\
0,\left(h_{s}, h_{g}\right) \in B .
\end{array}\right.
$$

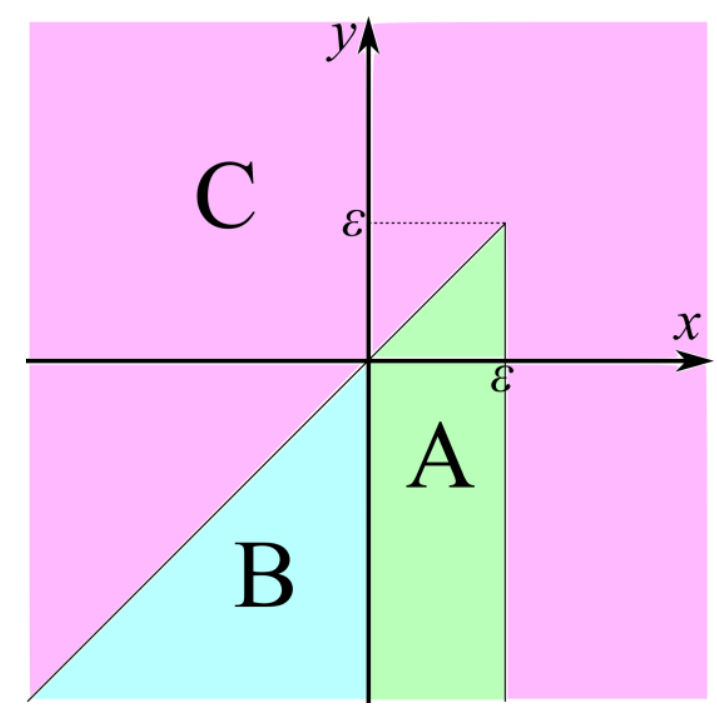

Figure 1. Decomposition of $h_{s}$ and $h_{g}$ range 


\section{NUMERICAL SOLUTION}

The system to be solved is composed of coupled surface flow equations (1) and subsurface flow equations (5). We use finite volume method and implicit Euler scheme to discretize model equations. Newton-Raphson method with relaxation is applied to solve the nonlinear problem in GeRa. Surface mesh on $\Gamma_{S}$ is obtained as trace of 3D mesh in $\Omega$.

To define the residual on every Newton-Raphson iteration $l$ we decompose it into three parts. Consider first the residual $R_{s, i}^{l, n}$ of the surface flow equation in $i$-th cell $E_{i}$ of surface mesh in $\Gamma_{s}$ at $n$-th timestep:

$$
R_{s, i}^{l, n}=R_{a c c, s, i}^{l, n}+R_{f l o w, s, i}^{l, n}+R_{s s, s, i}^{l, n}
$$

Here accumulation term $R_{a c c, s, i}^{l, n}$ corresponds to time derivative and precipitation sources, flow term $R_{\text {flow }, s, i}^{l, n}$ corresponds to water flow inside the computational domain (i.e. $\Gamma_{s}$ for surface runoff) and surface-subsurface term $R_{s s, s, i}^{l, n}$ corresponds to surface-subsurface flux. The same approach is applied to calculate groundwater flow equation residual:

$$
R_{g, i}^{l, n}=R_{a c c, g, i}^{l, n}+R_{\text {flow }, g, i}^{l, n}+R_{s s, g, i}^{l, n} .
$$

Note that $R_{s, i}^{l, n}$ and $R_{g, i}^{l, n}$ are functions of both surface water depth and groundwater pressure head as surface-subsurface flux depends on both of these variables and we use fully implicit scheme.

The combination of two vectors $R_{s, i}^{l, n}$ and $R_{g, i}^{l, n}$ is a residual vector for Newton-Raphson method.

\subsection{Discretization of surface runoff model}

Accumulation term corresponding to time derivative and source term (precipitation) can be written as follows:

$$
R_{a c c, s, i}^{l, n}=S_{i} \frac{h_{s, i}^{l, n}-h_{s, i}^{n-1}}{\Delta t^{n}}-S_{i} q_{i}^{n}
$$

where $S_{i}$ is the area of $E_{i}, n$ is the time step index, $h_{s, i}^{l, n}$ is the surface water depth at $l$-th Newton-Raphson iteration in $E_{i}, \Delta t^{n}$ is the time increment, $q_{i}^{n}$ is the precipitation rate (or other sources) in $E_{i}$.

Flow term corresponds to water flow on the surface domain. Using linear two-point flux approximation, we get the following expression:

$$
R_{f l o w, s, i}^{l, n}=\sum_{e_{i j} \in \partial E_{i}} K_{s, i j}^{l, n} \frac{h_{s, j}^{n}-h_{s, i}^{n}}{\left|c_{i} c_{j}\right|}\left|l_{i j}\right|,
$$

where summation is over surface mesh cells neighboring to $E_{i}$ through edges, $K_{s, i j}^{l, n}$ is 
discretization of surface conductivity on a common edge $e_{i j}$ of cells $E_{i}$ and $E_{j},\left|c_{i} c_{j}\right|$ is the distance between $E_{i}$ and $E_{j}$ cells' centers, $\left|l_{i j}\right|$ is the length of $e_{i j}$.

$$
K_{s, i j}^{l, n}=\left\{\begin{array}{l}
\frac{\left(h_{s, i j}^{l, n}\right)^{5 / 3}}{v\left(\left(\nabla_{x} H_{s, i j}^{l, n}\right)^{2}+\left(\nabla_{y} H_{s, i j}^{l, n}\right)^{2}\right)^{1 / 4}}, h_{s, i j}^{l, n} \geq 0, \\
0, h_{s, i j}^{l, n}<0,
\end{array}\right.
$$

where $h_{s, i j}^{l, n}$ is approximation of $h_{s}$ on $e_{i j}$ at $l$-th Newton iteration at $n$-th timestep, $\left[\nabla_{x} H_{s, i j}^{l, n}, \nabla_{y} H_{s, i j}^{l, n}\right]$ is approximation of $\nabla H_{s}$ on $e_{i j}$ at $l$-th nonlinear iteration at $n$-th time step. For negative $h_{s, i j}^{l, n}, K_{s, i j}^{l, n}$ is assumed to be equal to zero.

We use upwind approximation for the numerator of (18):

$$
h_{s, i j}^{l, n}=\left\{\begin{array}{l}
h_{s, i}^{l, n}, H_{s, i}^{l, n} \geq H_{s, j}^{l, n} \\
h_{s, j}^{l, n}, H_{s, i}^{l, n}<H_{s, j}^{l, n},
\end{array}\right.
$$

where $z_{i}$ and $z_{j}$ are $z$-coordinates of $E_{i}$ and $E_{j}$ centers respectively. We also use upwind approximation for the denominator of (18). Assume, that $E_{k}$ is the upwind cell, i.e.

$$
E_{k}=\left\{\begin{array}{l}
E_{i}, H_{s, i}^{l, n} \geq H_{s, j}^{l, n}, \\
E_{j}, H_{s, i}^{l, n}<H_{s, j}^{l, n} .
\end{array}\right.
$$

For a cell $E_{k}$ consider two sets of cells. $\Sigma_{k}^{\text {edge }}$ is a set of surface mesh cells neighboring to $E_{k}$ over an edge, $\Sigma_{k}^{\text {node }}$ is a set of surface mesh cells neighboring to $E_{k}$ over a node. For example shown in fig. $2 \Sigma_{j}^{\text {edge }}=\left\{i, j_{1}^{\text {edge }}, j_{2}^{\text {edge }}\right\}, \Sigma_{j}^{\text {node }}=\Sigma_{j}^{\text {edge }} \cup\left\{j_{1}^{\text {node }}, j_{2}^{\text {node }}, j_{3}^{\text {node }}, \ldots, j_{9}^{\text {node }}\right\}$.

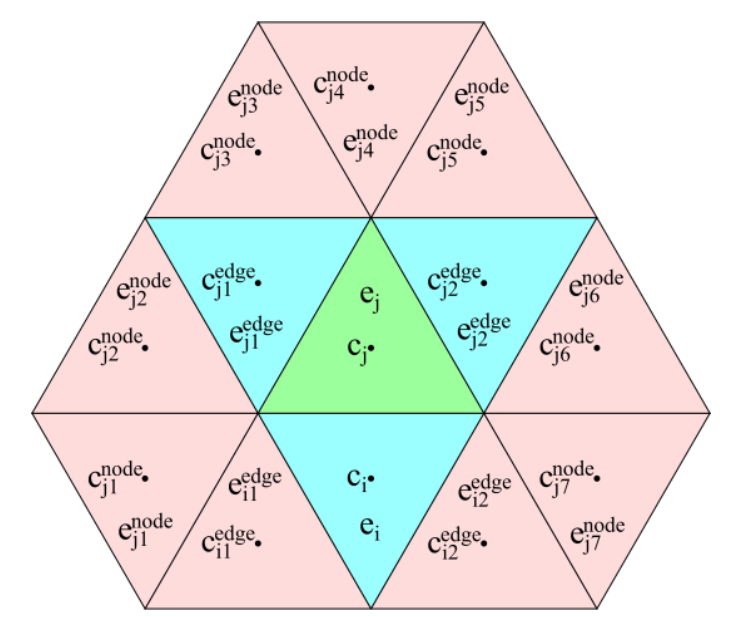

Figure 2. Illustration of $E_{j}^{\text {edge }}$ and $E_{j}^{\text {node }}$ sets for $j$-th cell, $E_{j}^{\text {edge }}$ consists of cyan-colored cells, $E_{j}^{\text {node }}$ consists of cyan and pink-colored cells 
For each element of $\Sigma_{k}^{\text {edge }}$ we consider the following equation based on Taylor series:

$$
H_{s, \alpha}^{l, n}=H_{s, k}^{l, n}+\left(x_{\alpha}-x_{k}\right) \nabla_{x} H_{s, k}^{l, n}+\left(y_{\alpha}-y_{k}\right) \nabla_{y} H_{s, k}^{l, n}
$$

where $\alpha$ is an element of $\Sigma_{k}^{e d g e}, x_{\gamma}, y_{\gamma}$ are $x, y$ coordinates of $\gamma$-th cell, $H_{s, k}^{l, n}=h_{s, k}^{l, n}+z_{k}$. Equations (21) for each $\alpha \in \Sigma_{k}^{e d g e}$ compose a linear system of equations for unknown $H_{s, x, k}^{l, n}$ and $H_{s, y, k}^{l, n}$. In case if this system is underdetermined we use $\Sigma_{k}^{n o d e}$ set of cells instead of $\Sigma_{k}^{e d g e}$.

Consider the following matrix and vector:

$$
A=\left[\begin{array}{cc}
x_{\alpha_{1}}-x_{k} & y_{\alpha_{1}}-y_{k} \\
x_{\alpha_{2}}-x_{k} & y_{\alpha_{2}}-y_{k} \\
x_{\alpha_{3}}-x_{k} & y_{\alpha_{3}}-y_{k} \\
\ldots & \ldots \\
x_{\alpha_{m}}-x_{k} & y_{\alpha_{m}}-y_{k}
\end{array}\right], \quad b=\left[\begin{array}{c}
H_{s, \alpha_{1}}^{l, n}-H_{s, k}^{l, n} \\
H_{s, \alpha_{2}}^{l, n}-H_{s, k}^{l, n} \\
H_{s, \alpha_{3}}^{l, n}-H_{s, k}^{l, n} \\
\ldots \\
H_{s, \alpha_{m}}^{l, n}-H_{s, k}^{l, n}
\end{array}\right],
$$

where $\alpha_{1}, \alpha_{2}, \alpha_{3}, \ldots, \alpha_{m}$ are elements of $\Sigma_{k}^{\text {edge }}$ or $\Sigma_{k}^{\text {node }}$ (depending on whether linear system $A x=b$ is underdetermined or not for $\Sigma_{k}^{e d g e}$ ).

The gradient $\left[\nabla_{x} H_{s, i j}^{l, n}, \nabla_{y} H_{s, i j}^{l, n}\right]$ is defined as argmin $\|A x-b\|^{2}$ (linear least squares problem solution):

$$
\left[\nabla_{x} H_{s, i j}^{l, n}, \nabla_{y} H_{s, i j}^{l, n}\right]^{T}=\left(A^{T} A\right)^{-1} A^{T} b
$$

\subsection{Discretization of groundwater flow model}

Again, consider separate components of nonlinear residual.

$$
R_{a c c, g, i}^{l, n}=V_{i}\left(\frac{\theta\left(h_{g, i}^{l, n}\right)-\theta\left(h_{g, i}^{n-1}\right)}{\Delta t^{n}}+S\left(h_{g, i}^{l, n}\right) s_{s t o r} \frac{h_{g, i}^{l, n}-h_{g, i}^{n-1}}{\Delta t^{n}}\right),
$$

where $V_{i}$ is the volume of $i$-th subsurface cell of $3 \mathrm{~d}$ mesh, $h_{g, i}^{l, n}$ is the groundwater pressure head at $l$-th nonlinear iteration at $n$-th time step in this cell.

$$
R_{f l o w, g, i}^{l, n}=\sum_{j} K_{g}\left(h_{g, i j}^{l, n}\right) \frac{h_{g, j}^{l, n}-h_{g, i}^{l, n}}{\left|c_{i} c_{j}\right|} S_{i j},
$$

where summation is over cells of subsurface mesh neighboring to $i$-th cell through a face, $\left|c_{i} c_{j}\right|$ is the distance between centers of $i$-th and $j$-th cells, $S_{i j}$ is the area of a common face of these cells, $K_{g}\left(h_{g}\right)$ is defined by (7), $h_{g, i j}^{l, n}$ is upwind pressure head defined by $h_{g, i j}^{l, n}=\max \left\{h_{g, i}^{l, n}, h_{g, j}^{l, n}\right\}$. 


\subsection{Discretization of surface-subsurface flux}

Consider residual term $R_{s s, s, i}^{l, n}$. We define this residual term as follows (domains $A, B$ and $C$ are depicted on fig. 1):

$$
R_{s s, s, i}^{l, n}=S_{i}\left\{\begin{array}{l}
\frac{K_{s s}}{d}\left(h_{s, i}^{l, n}-\overline{h_{g, i}^{l, n}}\right),\left(h_{s, i}^{l, n}, \overline{h_{g, i}^{l, n}}\right) \in C, \\
-\frac{K_{s s}}{d}\left(-\frac{1}{\varepsilon^{2}}\left(h_{s, i}^{l, n}\right)^{3}+\frac{1}{\varepsilon}\left(h_{s, i}^{l, n}\right)^{2}+\left(\overline{h_{g, i}^{l, n}}-\varepsilon\right) \frac{1-\cos \frac{\pi h_{s, i}^{l, n}}{\varepsilon}}{2}\right),\left(h_{s, i}^{l, n}, \overline{h_{g, i}^{l, n}}\right) \in A, \\
0,\left(h_{s, i}^{l, n}, \overline{h_{g, i}^{l, n}}\right) \in B .
\end{array}\right)
$$

The groundwater counterpart of this term can be defined by $R_{s s, g, i}^{l, n}=-R_{s s, s, i_{s}}^{l, n}$, where $i$ is an index of top level $3 \mathrm{~d}$ subsurface mesh cell, which has $i_{s}$-th $2 \mathrm{~d}$ surface mesh cell as one of its faces.

\section{NUMERICAL EXPERIMENTS}

Three numerical experiments are considered. In the first one no groundwater flow is modelled as we verify simple surface runoff model without coupling. The following two experiments are devoted to coupled surface-subsurface simulation. Coupled numerical experiments are then examined for parallel implementation efficiency.

\subsection{Surface runoff}

In this numerical experiment, we model surface runoff without coupling with groundwater. Numerical solution is compared to analytical solution of kinematic wave equation. Note that assumptions of diffusive wave approximations differ from kinematic wave. However, we propose to compare diffusive and kinematic wave approximation solutions due to the following arguments. First, analytical solutions for diffusive wave equation presented in papers are obtained using additional strong assumptions [33], [34]. Second, both approximations are formulated for the same original shallow water equations, thus approximate the same model.

Ground surface is a $200 \mathrm{~m} \times 100 \mathrm{~m}$ rectangle tilted with slope equal to 0.01 along the longest side. However, we add artificial river banks to prevent water outflow from the lateral sides of the domain. Geometry of the domain is illustrated by fig. 3. Rainfall intensity is equal to $5 \times 10^{-6} \mathrm{~m} / \mathrm{s}$ for the first 15000 seconds of the experiment and 0 for the next 15000 seconds of the experiment. Overall experiment duration is 30000 seconds. Manning roughness coefficient is $v=0.05 \mathrm{~s} / \mathrm{m}^{1 / 3}$. Comparison of the numerical results for linear discharge density through the outlet with the analytical solution is depicted on fig. 4 (linear discharge density is equal to the discharge divided by the outlet length, which is equal to $100 \mathrm{~m}$ ). As one can see on the figure numerical results are close to the analytical solution, however some qualitative difference remains. The latter may be caused by the slight diffusive and kinematic wave 
model disagreement.

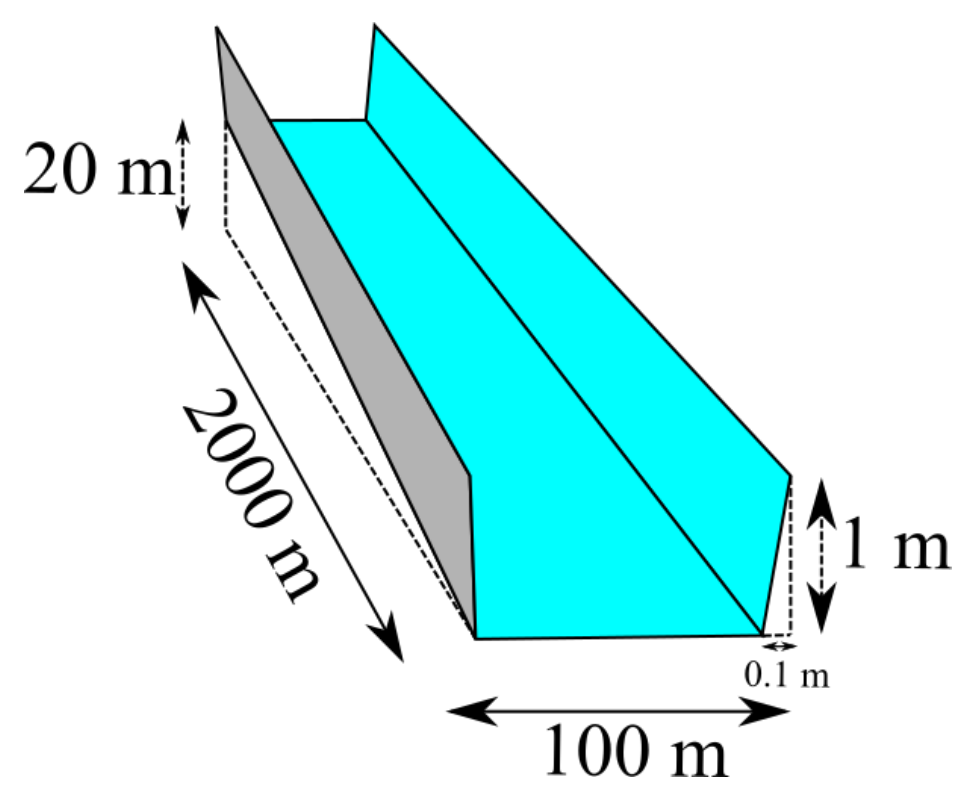

Figure 3. Geometry of tilted v-catchment numerical experiment domain

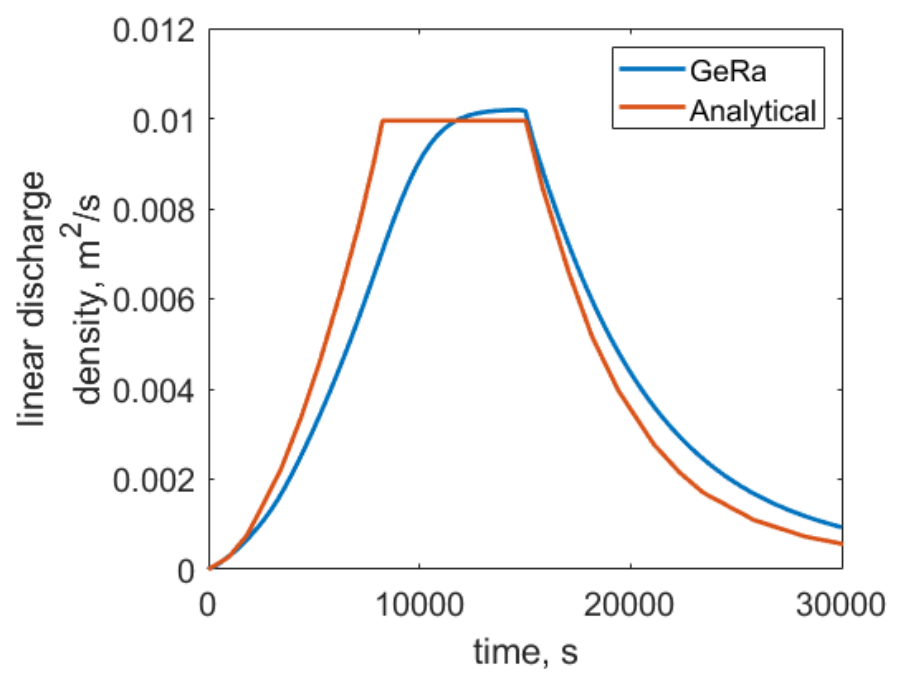

Figure 4. Water discharge dynamics for tilted v-catchment numerical experiment

\subsection{Tilted v-catchment with subsurface}

This numerical experiment as well as corresponding other simulators' numerical results are described in [4]. Ground surface is a $10 \mathrm{~m}$ wide channel with parallel walls with banks tilted in $x$ and $y$ directions. Slope in $y$ direction is constant and equal to 0.02 , slope in $x$ direction is zero for the channel, and 0.05 for channel banks. Bottom of the domain has the same geometry as ground surface and is located $5 \mathrm{~m}$ below the surface (see fig. 5 for domain geometry scheme). Two different precipitation scenarios were modeled: no rainfall during the 
120 hours of experiment in the first scenario, 20 hours of rainfall with precipitation rate 0.1 $\mathrm{m} / \mathrm{h}$ and 100 hours of recession in the second scenario. Authors of [4] suggest to use zero surface water depth and vertically hydrostatic initial conditions with water table $2 \mathrm{~m}$ below the ground surface as initial conditions. Boundary conditions are critical depth boundary condition for surface layer and no-flux boundary conditions for subsurface.

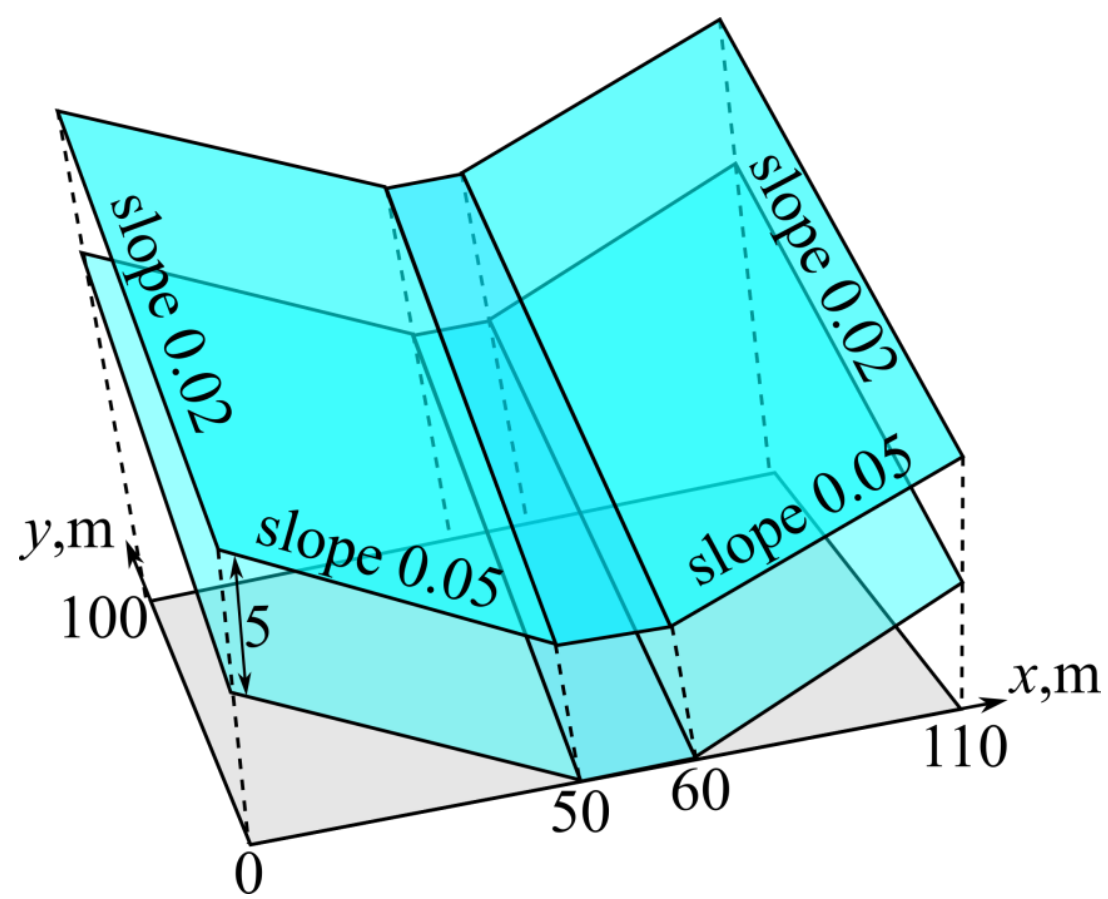

Figure 5. Geometry of tilted v-catchment numerical experiment domain

The following model parameters were used [4]:

- $\quad v=1.74 \times 10^{-3} \mathrm{~h} / \mathrm{m}^{1 / 3}$ for the channel and $v=1.74 \times 10^{-4} \mathrm{~h} / \mathrm{m}^{1 / 3}$ elsewhere,

- $K_{\text {sat }}=10 \mathrm{~m} / \mathrm{h}$,

$-n=2$ and $\alpha=6 \mathrm{~m}^{-1}$,

$-\theta_{r}=0.08, \theta_{s}=0.4$,

- $s_{\text {stor }}=10^{-5} \mathrm{~m}^{-1}$,

- precipitation rate: 0 for $120 \mathrm{~h}$ for the first scenario, $0.1 \mathrm{~m} / \mathrm{h}$ for the first $20 \mathrm{~h}$ and 0 afterwards for the second scenario.

One of the simulators considered in [4] uses first-order exchange as a coupling method (HGS simulator), however there is no exact value defined for proportionality coefficient for the surface-subsurface flux in this paper. Therefore, bottom sediment parameters were estimated for GeRa to fit the results of other simulators. For this numerical experiment, we used $K_{s s}=20 \mathrm{~m} /$ day and $d=0.2 \mathrm{~m}$.

Using these model parameters, we simulated the test case and obtained water dynamics for the surface and subsurface layers. Comparison discharge rate through the outlet obtained by GeRa code with other simulator results is presented in fig. 6 for the first scenario and fig. 7 for the second scenario. As one can see from the figures Gera software produces the solution 
close to the other simulators results. Absolute values of GeRa solution lie between other simulators.

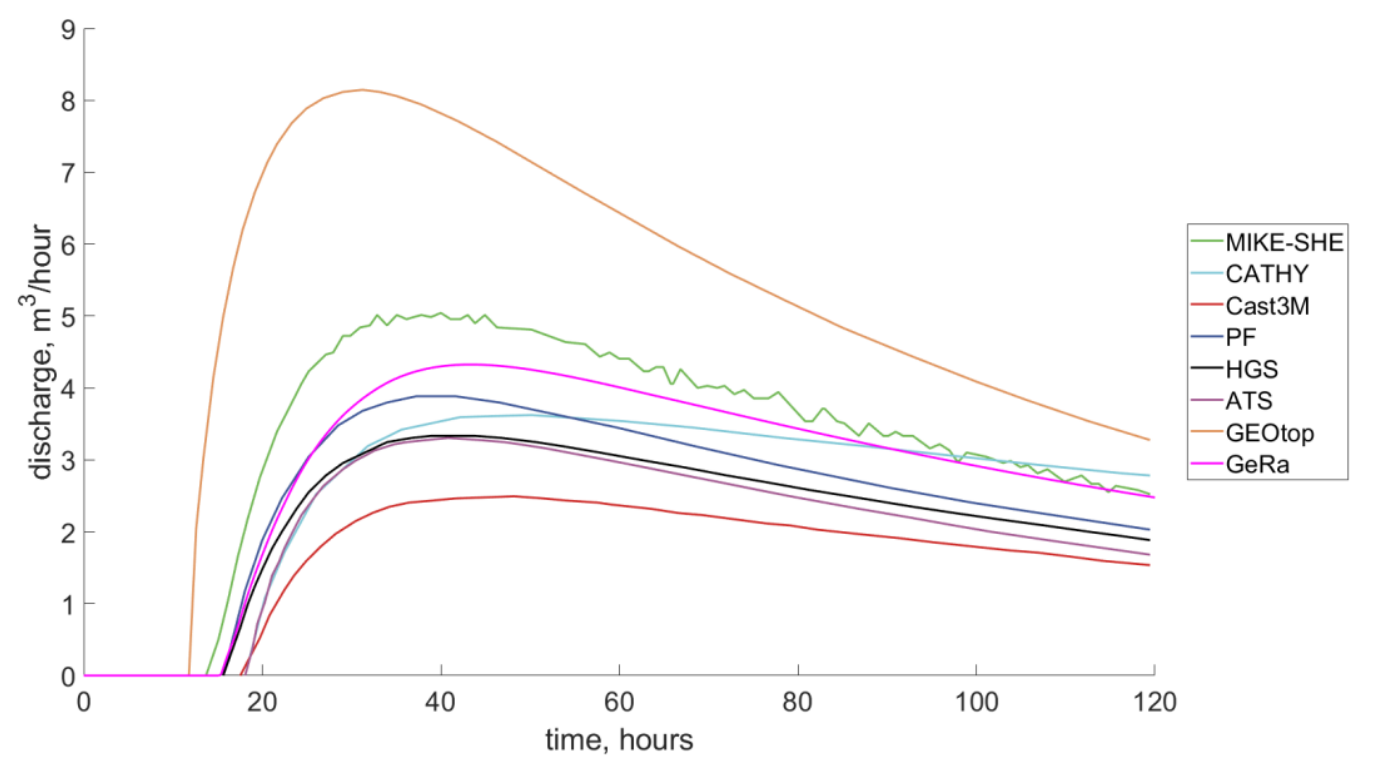

Figure 6. Water discharge dynamics for the first scenario of the tilted v-catchment benchmark

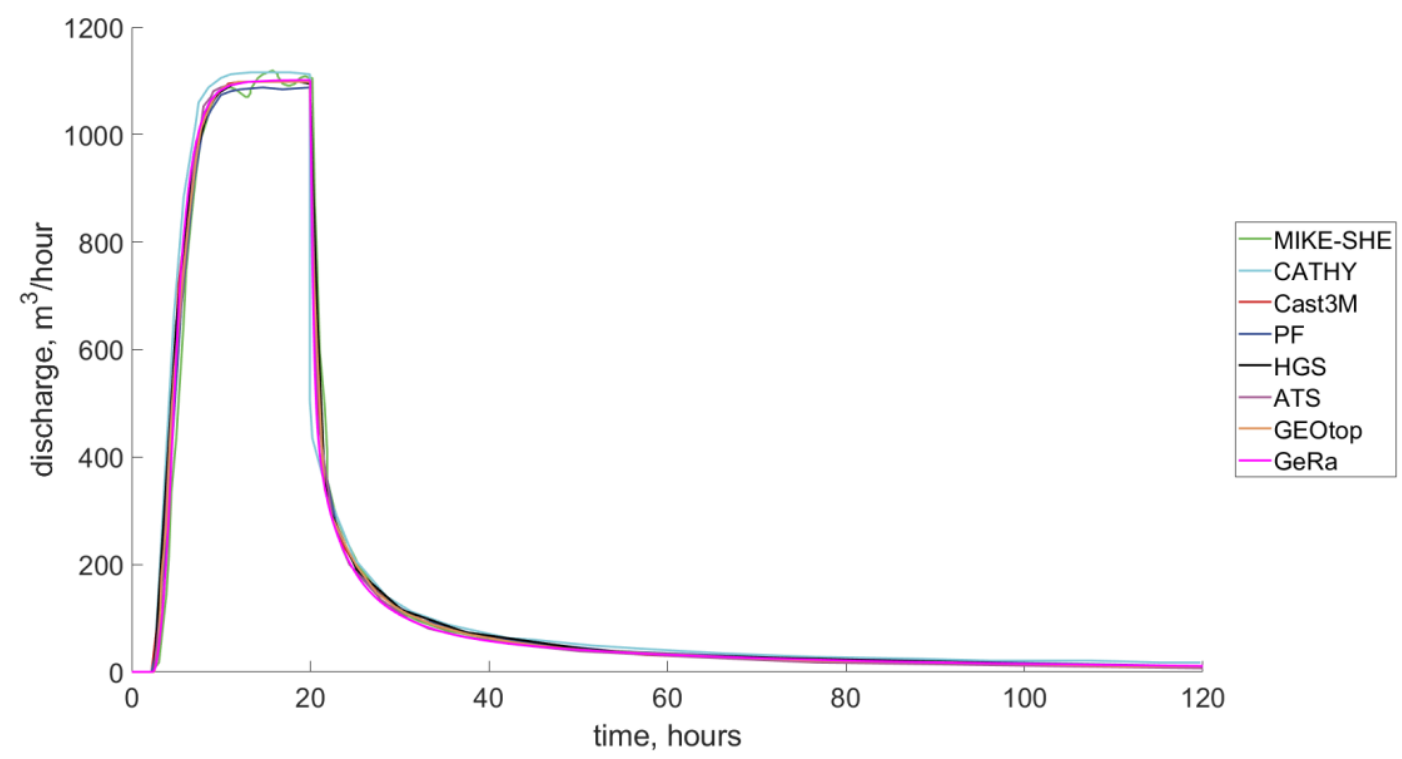

Figure 7. Water discharge dynamics for the second scenario of the tilted v-catchment benchmark

\subsection{Borden benchmark}

Field study was originally presented by Abdul and Gillham [35], [36] where outlet discharge has been measured for 100 minutes of the experiment. The experiment site is approximately $18 \mathrm{~m}$ wide and $90 \mathrm{~m}$ long. The exact surface geometry is described by Digital Elevation Model of the terrain [4] and is depicted in fig. 8. We considered a region with relief 
level less than $3.02 \mathrm{~m}$ as a channel domain and the rest of the surface as channel banks. The subsurface computational domain is bounded by $z=0$ plane at the bottom. Numerical experiment is implemented and described in [4], [20], [23].

We used the following model parameters:

$-v=0.03 \mathrm{~s} / \mathrm{m}^{1 / 3}$ for the channel and $v=0.3 \mathrm{~s} / \mathrm{m}^{1 / 3}$ elsewhere [20], [23],

- $K_{\text {sat }}=0.036 \mathrm{~m} / \mathrm{h} \mathrm{[4],}$

- $n=6$ and $\alpha=1.9 \mathrm{~m}^{-1}[4]$,

$-\theta_{r}=0.067, \theta_{s}=0.37[4]$,

- $s_{\text {stor }}=10^{-6} \mathrm{~m}^{-1}$,

- precipitation rate: $0.02 \mathrm{~m} / \mathrm{h}$ for the first 50 minutes, 0 for the last 50 minutes.

For this numerical experiment $K_{s s}=0.47 \mathrm{~m} /$ day and $d=0.2 \mathrm{~m}$ were used. Note that authors of [4] used constant value for Manning's roughness for the whole domain, while different values for the channel and the channel banks are used in [20], [23].

Zero water level on the surface and hydrostatic initial conditions with water table at $z=$ $2.78 \mathrm{~m}$ were used as initial conditions. Boundary conditions are critical depth boundary for the surface layer and no-flux boundary conditions for the subsurface.

Comparison between GeRa numerical discharge rate, other simulator discharge rate and experimental data is depicted in fig. 9. As one can see from the figure GeRa results are close to the experimental discharge rate. Moreover GeRa results agree with other simulators under consideration.

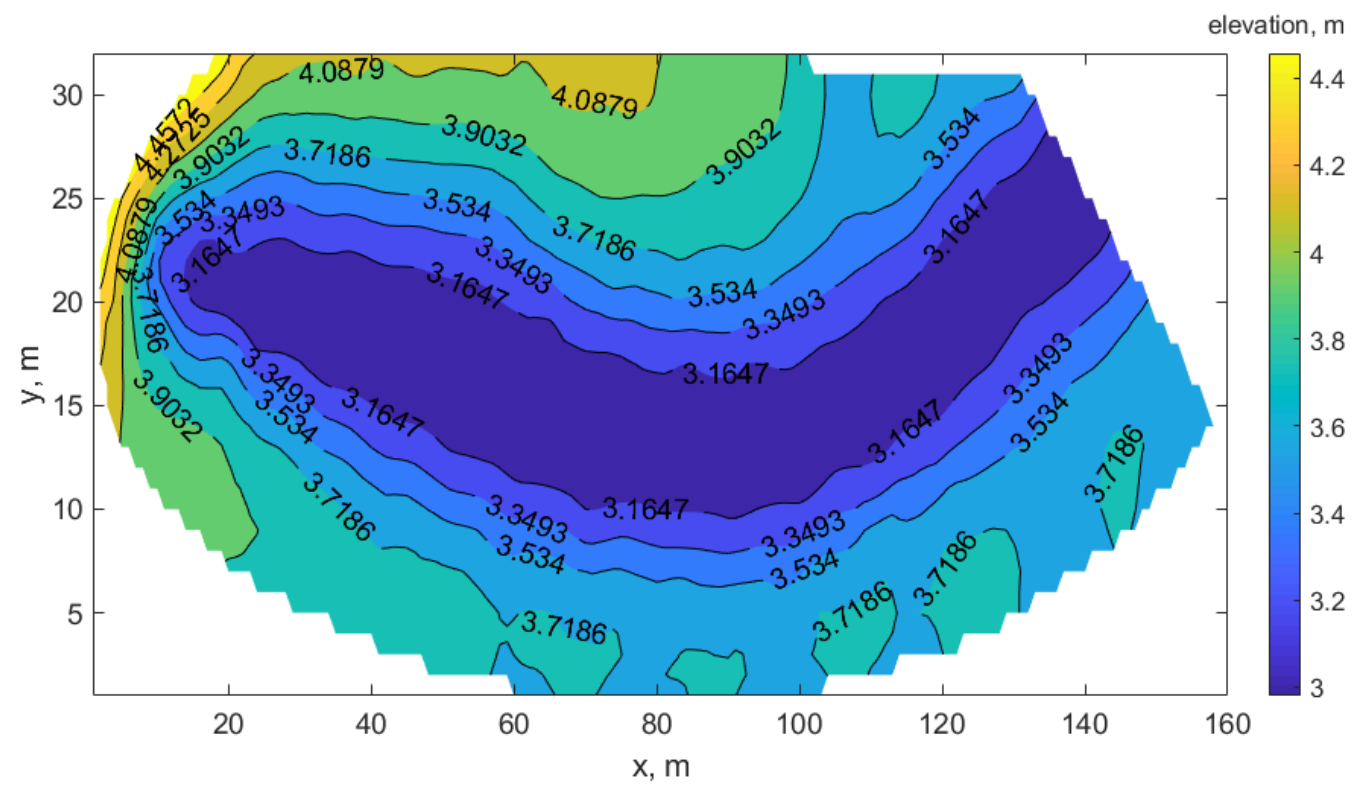

Figure 8. Borden benchmark surface elevation described by Digital Elevation Model with $0.5 \mathrm{~m}$ resolution [4] 


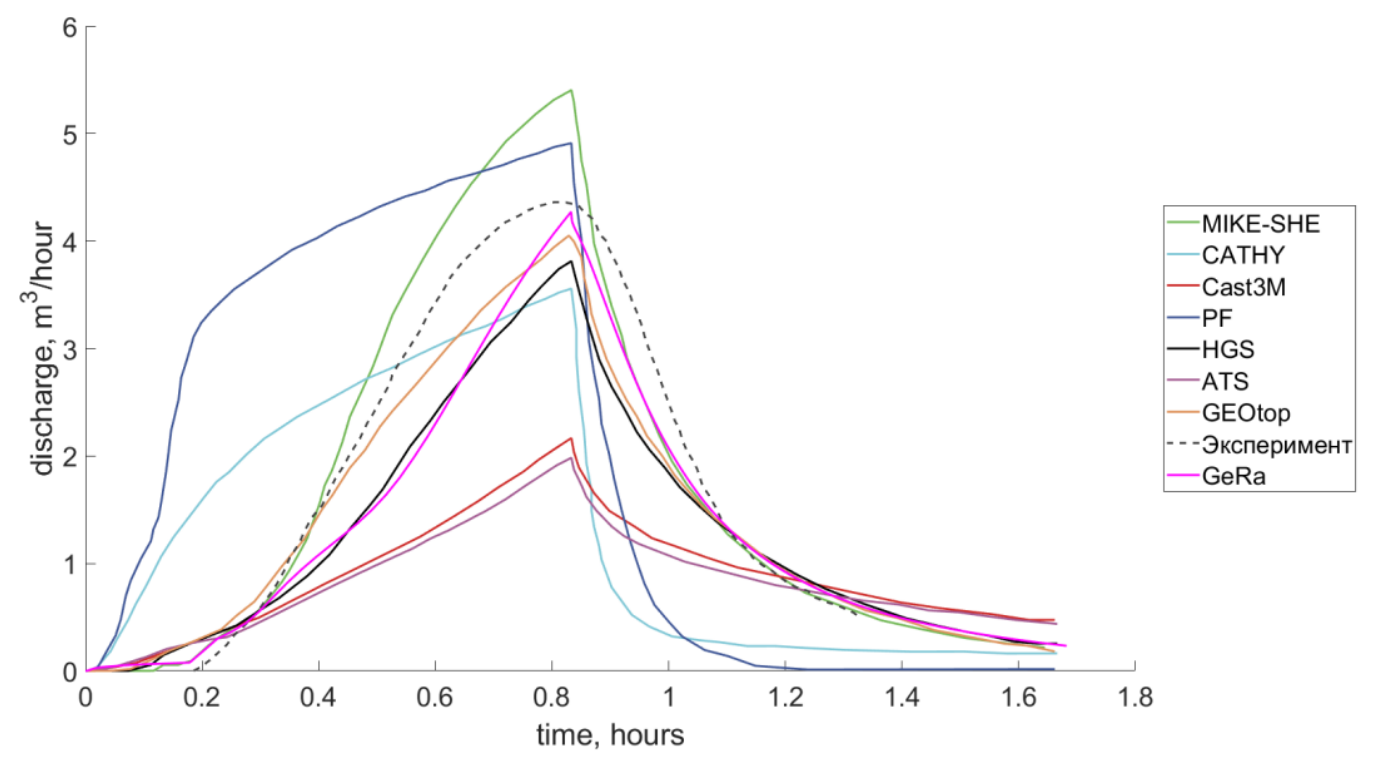

Figure 9. Water discharge dynamics for Borden benchmark

\subsection{Parallel numerical experiments}

In this section, we consider parallel efficiency of the coupled surface-subsurface model implemented in GeRa. Parallelization is implemented with MPI technology used in INMOST. For the numerical experiments presented in the section we dramatically refined the meshes for the experiments described previously. During Newton iteration, we need to solve a system of linear equations. Note, that in case of convergence failure we refine the time step. Maximum number of Newton iterations before the time step refinement is one of nonlinear solver parameters. To solve the linear systems of equations obtained on each Newton iteration we use PETSc package [37], namely BiCGStab solver with Schwartz preconditioner. On each processor ILU(k) preconditioner is used. For the mesh cell distribution between processors ParMETIS package is used [38].

All experiments are performed on INM RAS cluster [39] using the computational nodes of the $\mathrm{x} 12$ core segment:

- Compute Node Arbyte Alkazar+ R2Q50

- 24 cores (two 12-core Intel Xeon E5-2670v3@2.30GHz processors or Intel Xeon Silver 4214@2.20GHz);

- RAM: 64 GB;

- Operating system: SUSE Linux Enterprise Server 15 SP2;

- Network: Mellanox Infiniband.

Due to node configuration we consider not $1,2,4, \ldots, 2^{\mathrm{n}}$ cores, but $3,6, \ldots, 3^{*} 2^{\mathrm{n}}$ cores to measure parallel efficiency.

For the tilted v-catchment numerical experiment, (first precipitation scenario is considered) mesh size is 285750 cells. For Newton iterations, nonlinear problem parameters are the following:

- initial time step is 0.001 days;

- maximum number of nonlinear iterations before time step reduction is 40 ; 
- stopping criterion is residual reduction by $10^{-3}$ factor.

For linear system solution PETSc parameters are the following:

- Schwartz overlap between processors is 1;

- ILU factor level for each processor is 1 ;

- stopping criterion is initial residual reduction by factor $10^{-9}$;

In the Borden experiment, mesh size is 278140 cells. For Newton iterations, nonlinear problem parameters are the following:

- initial time step is 0.001 days;

- maximum number of nonlinear iterations before time step reduction is 300 ;

- stopping criterion is residual reduction by factor $10^{-4}$.

For linear system solutions PETSc parameters are the following:

- Schwartz overlap between processors is 3 ;

- ILU factor level for each processor is 3;

- Stopping criterion is initial residual reduction by factor $10^{-9}$.

Results are shown in the Table 1 for the tilted v-catchment experiment and Table 2 for the Borden experiment. For each number of processors (first column) we list total solution time of the experiment (second column), acceleration (third column) and efficiency of parallelization (fourth column). Acceleration is the ratio between total solution times for current number of processors and for the baseline number of processors. The baseline number is equal to 3 for tilted v-catchment benchmark and 12 for Borden benchmark (we do not use serial computation on a single processor due to long computational time for the refined mesh). Parallelization efficiency is the ratio between solution times for current number of processors and for two times smaller number of processors. In other words, efficiency value shows a speedup for one step of processors number increasing.

\begin{tabular}{|l|l|l|l|}
\hline $\begin{array}{l}\text { Number of } \\
\text { processors }\end{array}$ & Solution time & Acceleration & Efficiency \\
\hline 3 & 29456 & 1.0 & - \\
\hline 6 & 19521 & 1.5 & 1.5 \\
\hline 12 & 11039 & 2.7 & 1.8 \\
\hline 24 & 5081 & 5.8 & 2.2 \\
\hline 48 & 2645 & 11.1 & 1.9 \\
\hline 96 & 1267 & 23.4 & 2.1 \\
\hline 192 & 838 & 35.1 & 1.5 \\
\hline
\end{tabular}

Table 1. Parallel efficiency results for the tilted v-catchment experiment

\begin{tabular}{|l|l|l|l|}
\hline $\begin{array}{l}\text { Number of } \\
\text { processors }\end{array}$ & Solution time & Acceleration & Efficiency \\
\hline 12 & 92684 & 1.0 & - \\
\hline 24 & 51997 & 1.8 & 1.8 \\
\hline 48 & 28083 & 3.3 & 1.8 \\
\hline 96 & 15138 & 6.1 & 1.9 \\
\hline 192 & 9111 & 10.17 & 1.7 \\
\hline
\end{tabular}

Table 2. Parallel efficiency results for the Borden experiment 
Both experiments demonstrate good scalability and parallel efficiency of coupled surfacesubsurface water simulations. Maximum speedup is 35 times for tilted v-catchment experiment on 192 cores (theoretical maximum is 64 times). Both experiments also demonstrate fair efficiency. Average efficiency is more than 1.6 for both experiments and in some cases hyper linear speedup is observed.

\section{CONCLUSIONS}

Surface runoff model implemented in GeRa software package is described in the article. Verification benchmarks previously applied to the serial implementation of the model in [17] were used here to demonstrate validity of the parallel version of the model itself as well as coupled surface-subsurface model. We also used these benchmarks to assess parallelization efficiency of the coupled model. Numerical experiments show good scalability of the implementation. The acceleration for the parallel implementation is up to 35 times for 192 processors for the tilted $\mathrm{v}$-catchment benchmark relative to the baseline time obtained for 3 processors.

We also suggested surface-subsurface flux smoothing approach in order to prevent nonlinear solver oscillations.

\section{REFERENCES}

[1] I. Kapyrin, A. V. Ivanov, V. G. Kopytov, and S. S. Utkin, "Integral code GeRa for radioactive waste disposal safety validation", Gornyi Zhurnal, (10), 44-50 (2015).

[2] N. Collier, H. Radwan, L. Dalcin, and V.M. Calo, "Diffusive Wave Approximation to the Shallow Water Equations: Computational Approach", Procedia Computer Science, 4, 1828-1833 (2011).

[3] H. J. G. Diersch and P. Perroschet, "On the primary variable switching technique for simulating unsaturatedsaturated flows", Advances in Water Resources, 23(3), 271-301 (2011).

[4] S. Kollet, M. Sulis, R. M. Maxwell,C. Paniconi, M. Putti, G. Bertoldi, E. T. Coon, E. Cordano, S. Endrizzi, E. Kikinzon, E. Mouche, C. Mugler, Y.-J. Park, J. C. Refsgaard, S. Stisen, and E. Sudicky, "The integrated hydrologic model intercomparison project, IH-MIP2: A second set of benchmark results to diagnose integrated hydrology and feedbacks", Water Resources Research, 53(1), 867-890 (2017).

[5] J. E. Liggett, A. D. Werner, and C. T. Simmons, "Influence of the first-order exchange coefficient on simulation of coupled surface-subsurface flow", J. of Hydrology, 414-415, 503-515 (2012).

[6] R. Abdelaziz, Hai Ha Le, "MT3DMSP - A parallelized version of the MT3DMS code", J. of African Earth Sciences, 100, 1-6 (2014).

[7] D. Su, K.U. Mayer, K.T.B. MacQuarrie, "MIN3P-HPC: A High-Performance Unstructured Grid Code for Subsurface Flow and Reactive Transport Simulation", Math Geosci (2020).

[8] D. Yanhui, L. Guomin, "A parallel pcg solver for MODFLOW", Ground water, 47, 845-50 (2009).

[9] K. Zhang, Y.S. Wu, K. Pruess, "User's guide for TOUGH2-MP-a massively parallel 
version of the TOUGH2 code", doi: 10.2172/929425 (2008).

[10] S.S. Khrapov, A.V. Khoperskov, "Application of Graphics Processing Units for SelfConsistent Modelling of Shallow Water Dynamics and Sediment Transport", Lobachevskii J Math, 41, 1475-1484 (2020).

[11] S. L. Painter, E. T. Coon, A. L. Atchley, M. Berndt, R. Garimella, J. D. Moulton, D. Svyatskiy, and C. J. Wilson, "Integrated surface/subsurface permafrost thermal hydrology: Model formulation and proof-ofconcept simulations", Water Resources Research, 52(8), 6062-6067 (2016).

[12] D. Anuprienko, I. Kapyrin, "Nonlinearity continuation method for steady-state groundwater flow modeling in variably saturated conditions" https://arxiv.org/abs/2008.00730 (Accessed November 9, 2020)

[13] A. Litvinenko, D. Logashenko, R. Tempone, G. Wittum, D Keyes, "Solution of the 3D density-driven groundwater flow problem with uncertain porosity and permeability", Int J Geomath, 11, 10 (2020). https://doi.org/10.1007/s13137-0200147-1.

[14] I. Konshin, I. Kapyrin, "Scalable Computations of GeRa Code on the Base of Software Platform INMOST", International Conference on Parallel Computing Technologies, 433-445 (2017).

[15] D. Bagaev, F. Grigoriev, I. Kapyrin, I. Konshin, V. Kramarenko, A. Plenkin, "Improving Parallel Efficiency of a Complex Hydrogeological Problem Simulation in GeRa", In book: Supercomputing, (2019). 10.1007/978-3-030-36592-9_22

[16] INMOST - A toolkit for distributed mathematical modeling. https://journals.aps.org/revtex (Accessed November 6, 2020).

[17] K. Novikov, I. Kapyrin, "Coupled Surface-Subsurface Flow Modelling Using the GeRa Software”, Lobachevskii Journal of Mathematics, 41, 538-551 (2020).

[18] S. Endrizzi, S. Gruber, M. Dall'Amico, and R. Rigon, "GEOtop 2.0: simulating the combined energy and water balance at and below the land surface accounting for soil freezing, snow cover and terrain effects", Geoscientific Model Development, 7(6), 2831-2857 (2014).

[19] R. Rigon, G. Bertoldi, and T. M. Over, "GEOtop: A Distributed Hydrological Model with Coupled Water and Energy Budgets”, J. of Hydrometeorology, 7(3), 371-388 (2006).

[20] HGS User Manual. https://www.aquanty.com/hgs-download (Accessed November 6, 2019).

[21] ParFlow User's Manual. https:/github.com/parflow/parflow/blob/v3.6.0/parflowmanual.pdf (Accessed November 6, 2019).

[22] J. E. VanderKwaak and K. Loague, "Hydrologic-response simulations for the R-5 catchment with a comprehensive physics-based model", Water Resources Research, 37(4), 999-1013 (2001).

[23] J. E. VanderKwaak, Ph.d. diss. Numerical Simulation of Flow and Chemical Transport in Integrated Surface-Subsurface Hydrologic Systems, University of Waterloo, Waterloo, Ontario, Canada (1999).

[24] H. An and S. Yu, "Finite volume integrated surface-subsurface flow modeling on nonorthogonal grids", Water Resources Research, 50(3), 2312-2328 (2014).

[25] J.-O. Delfs, Ph.d. diss. An Euler-Lagrangian concept for transport processes in coupled hydrosystems, University of Tubingen, Tubingen, Germany (2010). 
[26] J.-O. Delfs, C.-H. Park, and O. Kolditz, "A sensitivity analysis of Hortonian flow", Advances in Water Resources, 32, 1386-1395 (2009).

[27] S.Weill, E. Mouche, and J. Patin, "A generalized Richards equation for surface/subsurface flow modelling", J. of Hydrology, 366(1), 9-20 (2009).

[28] M. Camporese, C. Paniconi, M. Putti, and S. Orlandini, "Surface-subsurface flow modeling with path-based runoff routing, boundary condition-based coupling, and assimilation of multisource observation data", Water Resources Research, 46(2), (2010).

[29] MIKE-SHE. Volume 2: Reference Guide. http://manuals.mikepoweredbydhi.help/2017/Water_Resources/MIKE_SHE_Printed_ V2.pdf. (Accessed November 6, 2019).

[30] M. Van Genuchten, "A Closed-form Equation for Predicting the Hydraulic Conductivity of Unsaturated Soils", Water Soil Science Society of America, 44(5), 892-898 (1980).

[31] Y. Mualem, "A new model for predicting the hydraulic conductivity of unsaturated porous media", Water Resources Research, 12(3), 513-522 (1976).

[32] B. A. Ebel, B. B. Mirus, C. S. Heppner, J. E. VanderKwaak, and K. Loague, "Firstorder exchange coefficient coupling for simulating surface water-groundwater interactions: parameter sensitivity and consistency with a physics-based approach", Hydrological Processes, 23(13), 1949-1959 (2009).

[33] Ping-Cheng Hsieh, Ding-You Wang, Ming-Chang Wu, "Analytical solution to a diffusion wave equation with variable coefficients for overland flow", Journal of Hydrology, 577, 123925 (2019). https://doi.org/10.1016/j.jhydrol.2019.123925.

[34] C.M. Kazezyılmaz-Alhan, Jr. M.A. Medina, "Kinematic and Diffusion Waves: Analytical and Numerical Solutions to Overland and Channel Flow", Journal of Hydraulic Engineering, 133(2), (2007).

[35] A. S. Abdul, Ph.d. diss. Experimental and Numerical studies of the effect of the capillary fringe on streamflow generation, University of Waterloo, Waterloo, Ontario, Canada (1985).

[36] A. S. Abdul and R. W. Gillham, "Field studies of the effects of the capillary fringe on streamflow generation", J. of Hydrology, 112(1-2), 1-18 (1989).

[37] PETSc, Suite of data structures and routines for the scalable (parallel) solution of scientific applications modeled by partial differential equations. https://www.mcs.anl.gov/petsc/index.html (Accessed: November 09, 2020)

[38] ParMETIS - Parallel graph partitioning and fill-reducing matrix ordering. http://glaros.dtc.umn.edu/gkhome/metis/parmetis/overview (Accessed: November 09, 2020)

[39] INM RAS cluster http://cluster2.inm.ras.ru (Accessed: November 09, 2020)

Received October 10, 2020 